\title{
Self Diffusion and Viscoelasticity in Entangled Systems I. Self-Diffusion Coefficients
}

\author{
Hiroshi FuJITA and Yoshiyuki EINAGA \\ Department of Macromolecular Science, Osaka University, \\ Toyonaka, Osaka 560. Japan
}

(Received April 2, 1985)

\begin{abstract}
Polymer self-diffusion coefficient $D_{\mathrm{s}}$ in entangled systems is formulated by postulating the free chain model and estimating the noodle effect on the basis of three assumptions. Here, the noodle effect refers to the frictional resistance exerted on a diffusing chain by its surrounding chains. For highly entangled solutions of a monodisperse polymer, $D_{\mathrm{s}}$ corrected for the segment friction coefficient is shown to vary linearly with $c_{\mathrm{p}}^{-1.5} M^{-2.5}$, where $c_{\mathrm{p}}$ and $M$ are the mass concentration and molecular weight of the polymer, respectively. The corresponding results for binary blends of homologous monodisperse polymers are compared with some published data, and a fairly good agreement is found between theory and experiment.
\end{abstract}

KEY WORDS Self Diffusion / Entangled System / Reptation / Free Chain

Model / Noodle Effect / Concentration Dependence / Molecular Weight

Dependence /

The reptation chain theory of de Gennes ${ }^{1}$ predicts that the self-diffusion coefficient $D_{\mathrm{s}}$ of a linear flexible polymer in entangled systems is inversely proportional to $M^{2}$, where $M$ is the molecular weight of the polymer. In recent years, many experimental data in support of this prediction have been published, ${ }^{2}$ and have strengthened the view that polymer chains in melt or in concentrated solution diffuse predominantly in the reptation mode. However, if these published data are examined carefully, it is found that few of them are actually concerned with systems in which polymer chains are sufficiently entangled with one another that the basic assumption of reptation theory is satisfied. Thus, it seems hasty to conclude that the reptation theoretical prediction $D_{\mathrm{s}} \propto M^{-2}$ has been amply confirmed by experiment. No theory is yet available to explain why the relation $D_{\mathrm{s}} \propto M^{-2}$ is observed in systems where polymer chains are virtually unentangled or nearly entangled.

In a recent paper, ${ }^{3}$ we have reanalyzed some published $D_{\mathrm{s}}$ data on entangled and unentangled polymer systems and inferred that the molecular weight dependence of $D_{\mathrm{s}}$ for a linear flexible polymer at fixed temperature and concentration varies from $D_{\mathrm{s}} \propto M^{-1}$ to $D_{\mathrm{s}} \propto M^{-2.5}$ as $M$ increases from below to above $M_{\mathrm{c}}$, where $M_{\mathrm{c}}$ is the molecular weight for the onset of entanglement effect on steady-state viscosity $\eta_{0}$. At present, the available data for $D_{\mathrm{s}}$ in the region of $M$ above $M_{\mathrm{c}}$ are very limited and, in addition, they do not cover sufficiently entangled regions because of the technical difficulties of measuring very low $D_{\mathrm{s}}$ (smaller than say $10^{-12} \mathrm{~cm}^{2} \mathrm{~s}^{-1}$ ). Therefore, the exponent -2.5 inferred from our data analysis may not be quite correct, but we are convinced that the $M$ dependence of $D_{\mathrm{s}}$ in well-entangled regions should be stronger than predicted by reptation theory. The purpose of the present paper is to formulate a simple theory of self diffusion which leads to a prediction consistent with this conviction.

In reptation theory, it is assumed that, 
owing to the topological constraints of entangled chains, the primitive chain-a coarsegrained polymer molecule - is forced to move as a whole along its contour, i.e., to reptate. For this motion to occur it is necessary that all segments of the primitive chain slide cooperatively and simultaneously in one curvilinear direction. Our basic question is whether entangled chains, forming a temporary network with relatively large meshes, can be a topological constraint strong enough to suppress any other motions of the chain than reptation in the region of large timescale. For a sufficiently flexible polymer it is hard to consider that such suppression occurs, since it is the basic property of flexible polymer that its segments (precisely, Kuhn's segments) have virtually no orientation correlation and tend to give the chain conformations as random as possible. Chain entanglements fluctuate in space and disappear and reform with time. This should weaken the topological constraints of entanglements to chain motion.

We suspect that the chains in flexible polymer concentrates do not suffer as strong topological constraints of entanglements as those assumed in the reptation theory, but can wriggle rather freely and isotropically as if they were in dilute solution, except for the following important point: in entangled systems, moving chains induce movement of their surrounding chains through interactions at the entangling points and bring about a loss of considerable energy. In this paper, such a coupling phenomenon is referred to as "noodle effect." Despite its importance in polymer dynamics, this effect has been almost completely ignored in recent theoretical studies on entangled polymer systems.

In the following, we develop a theory for $D_{\mathrm{s}}$, assuming the free chain model (see below) for polymer Brownian motion and formulating the noodle effect on some intuitive assumptions and approximations.

\section{THEORY}

\section{Monodisperse Systems}

First, we concern ourselves with a solution consisting of a pure solvent and a monodisperse polymer whose chain length (the number of segments) is $N$. It is assumed that $N$ and polymer mass concentration $c_{\mathrm{p}}$ are so high that the average length $N_{\mathrm{e}}$ between adjacent entangling points is sufficiently small in comparison with $N$. Thus, our formulation deals mathematically with asymptotic relations valid for $N / N_{\mathrm{e}} \gg 1$. This condition is explicitly used in the subsequent development.

Adopting the free chain model means assuming that the translational friction coefficient experienced by a chain diffusing in a solution is given by the product of $N$ and the average segment friction coefficient $\zeta$. Thus, for this model, $D_{\mathrm{s}}$ is represented by

$$
D_{\mathrm{s}}=k T(N \zeta)^{-1}
$$

where $k$ is the Boltzmann constant and $T$ the absolute temperature. We denote by $\zeta_{0}$ the friction coefficient of a "free" segment, i.e., one not entangled by any other chain, and by $\zeta_{\mathrm{e}}$ the additional friction coefficient per "entangled" segment which arises from noodle effect. Since the number of entangled segments per chain is $N / N_{\mathrm{e}}$ (under the condition $N_{\mathrm{e}}$ $\ll N$ ), it can be shown that $\zeta$ is given by

$$
\zeta=\zeta_{0}+\zeta_{\mathrm{e}} / N_{\mathrm{e}}
$$

It is reasonable to treat $\zeta_{0}$ as independent of $N$.

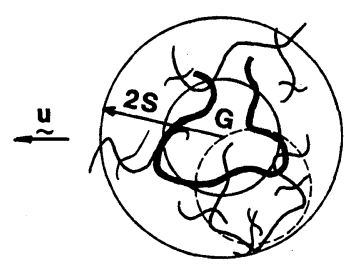

Figure 1. Model of entangled polymer chains. The heavy line is the central chain $\mathrm{C}$ moving with a speed $u$ relative to its surroundings. The larger circle defines the edge of the effective velocity field around $\mathrm{C}$. The dashed circle indicates the spherical domain occupied by the segments of a primary entangling chain. 
To estimate $\zeta_{\mathrm{e}}$ we consider what happens when the thick chain $\mathrm{C}$ (referred to as the central chain) in Figure 1 moves translationally at a velocity $\boldsymbol{u}$. Thin chains surrounding $\mathrm{C}$ must displace cooperatively to open space for $\mathrm{C}$ and receive frictional resistances from their surroundings. The noodle effect is concerned with the force needed to overcome these resistances.

\section{Assumption 1}

On statistical average, the velocity field $\mathrm{E}$ induced around $\mathrm{C}$ is considered spherically symmetric about the center of mass $\mathrm{G}$ of $\mathrm{C}$. Its radius may be of the order of $\mathrm{S}$, the root-meansquare radius of gyration of $\mathrm{C}$; segments situated far distant from $G$ need not accommodate themselves to the movement of $\mathrm{C}$. We assume the radius of $\mathrm{E}$ to be $2 S$, and refer to $\mathrm{E}$ with this radius as the effective velocity field of the central chain. Note that $\mathrm{S}$ is proportional to $N^{1 / 2}$, because excluded-volume effects vanish in polymer concentrates.

\section{Assumption 2}

We denote by $\boldsymbol{F}$ the force in the $\boldsymbol{u}$ direction that has to be applied to $G$ in order to overcome the frictional resistances experienced by the chains moving with $\mathrm{C}$. If the free drainage approximation is used, $\boldsymbol{F}$ may be expressed by

$$
\boldsymbol{F}=\zeta_{0} \int_{\mathrm{E}} \rho_{\mathrm{e}} \overline{\boldsymbol{u}} \mathrm{d} v
$$

where $\rho_{\mathrm{e}}$ is the average density of segments in a volume element $\mathrm{d} v$ which move cooperatively with $\mathrm{C}$ and $\overline{\boldsymbol{u}}$ the average velocity of these coupled segments in the $\boldsymbol{u}$ direction. We may visualize that, on statistical average, both $\rho_{\mathrm{e}}$ and $\overline{\boldsymbol{u}}$ decrease monotonically as we go from $G$ toward the edge of the field $\mathrm{E}$, where they vanish.

Since $\overline{\boldsymbol{u}}$ is proportional to $\boldsymbol{u}$, we approximate it by $K \boldsymbol{u}$, with $0<K<1$, and treat $K$ as independent of $N$ and $c_{\mathrm{p}}$ as well as of a position inside the field $\mathrm{E}$. Then, eq 3 becomes

$$
\boldsymbol{F}=\zeta_{0} K \boldsymbol{u} \int_{\mathrm{E}} \rho_{\mathrm{e}} \mathrm{d} v
$$

We approximate the integral in this equation by $n s$, with $n$ the number of chains directly entangling with $\mathrm{C}$ (such chains are referred to as primary entangling chains) and $s$ the number of segments per such chain which have substantial coupling with $\mathrm{C}$ and are found inside the field $\mathrm{E}$.

The spherical domain occupied by eachprimary entangling chain contains $\left(N / N_{\mathrm{e}}\right)^{3 / 2} N_{\mathrm{e}}$ segments (under the condition $N_{\mathrm{e}} \ll N$ ) on statistical average. According to Assumption 1, all these segments are situated inside E, regardless of the point at which the primary entangling chain entangles with $\mathrm{C}$. In fact, in Assumption 1, the radius of $\mathrm{E}$, i.e., $2 S$ was chosen so that this condition is satisfied. Though ad hoc, we assume that $s$ is proportional to $\left(N / N_{\mathrm{e}}\right)^{3 / 2} N_{\mathrm{e}}$ and that the proportionality factor $K^{\prime}$ is independent of $N$ and $c_{\mathrm{p}}$. This assumption for $s$ takes approximately into account the fact that part of the chains not directly entangling with $\mathrm{C}$ is dragged accompanying the movement of C.

With the approximations made above, eq 4 yields

$$
\boldsymbol{F}=\zeta_{0} n \boldsymbol{u} f N^{3 / 2} N_{\mathrm{e}}^{-1 / 2}
$$

where $f=K K^{\prime}$ is a parameter independent of $N$ and $c_{\mathrm{p}}$. Since $\zeta_{\mathrm{e}}$ is given by $\boldsymbol{F} / \boldsymbol{u n}$, we obtain

$$
\zeta_{\mathrm{e}}=\zeta_{0} f N^{3 / 2} N_{\mathrm{e}}^{-1 / 2}
$$

Substituting this into eq 2 yields

$$
\zeta=\zeta_{0}\left[1+f\left(N / N_{\mathrm{e}}\right)^{3 / 2}\right]
$$

which is inserted into eq 1 to give

$$
D_{\mathrm{s}}^{-1}=\zeta_{0}(k T)^{-1}\left[1+f\left(N / N_{\mathrm{e}}\right)^{3 / 2}\right] N
$$

For $N / N_{\mathrm{e}} \gg 1$ this tends asymptotically to the form

$$
D_{\mathrm{s}}^{-1}=f \zeta_{0}(k T)^{-1} N_{\mathrm{e}}^{-3 / 2} N^{5 / 2}
$$

Thus, $D_{\mathrm{s}}$ of a monodisperse polymer in highly entangled solutions should become proportional to $M^{-2.5}$ (here, $M$ is the molecular weight of the polymer) with increasing $M$, as we have inferred in our recent data analysis. ${ }^{3}$ 


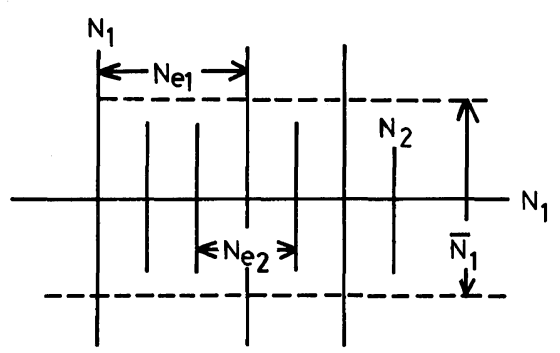

Figure 2. Definition of the average entanglement spacings $N_{\mathrm{e} 1}, N_{\mathrm{e} 2}$, and the average length $\bar{N}_{1}$ of the primary entangling chains for chain 1.

\section{Binary Blend Systems}

Next, we consider a solution consisting of a pure solvent and a blend of two homologous monodisperse polymers 1 and 2 whose chain lengths are $N_{1}$ and $N_{2}$, respectively. We assume $N_{2}<N_{1}$ and restrict ourselves to the case $N_{\mathrm{e}} \ll N_{2}$, where $N_{\mathrm{e}}$ has the same meaning as above.

We focus on a chain of polymer 1 (referred to as chain 1) in the solution. This chain is entangled by chains of both polymers 1 and 2 . As illustrated in Figure 2, the average chain length between its adjacent segments both entangled by polymer 1 chains is denoted by $N_{\mathrm{e} 1}$, and the corresponding quantity defined by entanglement of polymer 2 chains by $N_{\mathrm{e} 2}$. Then, we have the relation (under the condition $N_{\mathrm{e}} \ll N_{2}$ )

$$
1 / N_{\mathrm{e}}=1 / N_{\mathrm{e} 1}+1 / N_{\mathrm{e} 2}
$$

As before, the chains directly entangling with chain 1 are referred to as the primary entangling chains for chain 1. It can be shown that their average length $\bar{N}_{1}$ is expressed by

$$
\bar{N}_{1}=N_{\mathrm{e}}\left(N_{1} / N_{\mathrm{e} 1}+N_{2} / N_{\mathrm{w} 2}\right)
$$

\section{Assumption 3}

Chain 1 undergoes the same noodle effect from its surrounding chains as it does when diffusing through a network with mesh length $N_{\mathrm{e}}$ formed by a monodisperse polymer of chain length $\bar{N}_{1}$. This is a kind of mean field approximation.

It can be shown that $\bar{N}_{1}<N_{1}$ for $N_{2}<N_{1}$. Therefore, the segments contained in the spherical domain occupied by any primary entangling monodisperse chain of length $\bar{N}_{1}$ are all found inside the effective velocity field $E_{1}$ of chain 1. Thus, using the argument made in relation to Assumption 2, we obtain

$$
\zeta_{\mathrm{e} 1}=f \zeta_{0} \bar{N}_{1}{ }^{3 / 2} N_{\mathrm{e}}{ }^{-1 / 2}
$$

where $\zeta_{\mathrm{e} 1}$ denotes $\zeta_{\mathrm{e}}$ for polymer 1 . Substituting eq 10 and 11 yields

$$
\begin{aligned}
\zeta_{\mathrm{e} 1}= & f \zeta_{0} N_{\mathrm{e}}^{-1 / 2}\left[N_{1} N_{\mathrm{e}} / N_{\mathrm{e} 1}\right. \\
& \left.+N_{2}\left(1-N_{\mathrm{e}} / N_{\mathrm{e} 1}\right)\right]^{3 / 2}
\end{aligned}
$$

The average segment coefficient $\zeta_{1}$ for polymer 1 can be obtained by inserting eq 13 into

$$
\zeta_{1}=\zeta_{0}+\zeta_{\mathrm{e} 1} / N_{\mathrm{e}}
$$

which is valid under the assumed condition $N_{\mathrm{e}} \ll N_{1}$. The self-diffusion coefficient $D_{\mathrm{s} 1}$ of polymer 1 is given by

$$
D_{\mathrm{s} 1}=k T /\left(N_{1} \zeta_{1}\right)
$$

With eq 14 introduced into eq 15 , we obtain the asymptotic form of $D_{\text {s1 }}$ valid for $N_{\mathrm{e}} \ll N_{1}$ as follows:

$$
\begin{aligned}
D_{\mathrm{s} 1}^{-1}= & f \zeta_{0}(k T)^{-1} N_{\mathrm{e}}^{-3 / 2} N_{1}\left[N_{1} N_{\mathrm{e}} / N_{\mathrm{e} 1}\right. \\
& \left.+N_{2}\left(1-N_{\mathrm{e}} / N_{\mathrm{e} 1}\right)\right]^{3 / 2}
\end{aligned}
$$

where eq 13 has been substituted for $\zeta_{1}$.

It can be shown that $\bar{N}_{2}>N_{1}$ for $N_{2}<N_{1}$, where $\bar{N}_{2}$ is the average length of the chains directly entangling with chain 2 . Hence, on statistical average, some of the segments contained in the spherical domain occupied by each primary entangling monodisperse chain of length $\bar{N}_{2}$ appear outside the effective velocity field $\mathrm{E}_{2}$ of chain 2. According to Assumptions 1 and 2, such segments must be considered not to contribute to the noodle effect experienced by chain 2. It follows from Assumption 1 that their fraction in any primary entangling monodisperse chain for chain 2 is $1-\left(N_{2} / \bar{N}_{2}\right)$. 
From the above consideration we find that $\zeta_{\mathrm{e} 2}$, i.e., $\zeta_{\mathrm{e}}$ for polymer 2 , is given by

$$
\zeta_{\mathrm{e} 2}=f \zeta_{0} N_{2}{ }^{3 / 2} N_{\mathrm{e}}{ }^{-1 / 2}
$$

With this, we obtain

$$
D_{\text {s2 }}{ }^{-1}=f \zeta_{0}(k T)^{-1} N_{\mathrm{e}}{ }^{-3 / 2} N_{2}{ }^{5 / 2}
$$

as the asymptotic form of the self-diffusion coefficient $D_{\text {s2 }}$ of polymer 2 valid for $N_{\mathrm{e}} \ll N_{2}$. This gives an interesting prediction that. $D_{\mathrm{s} 2}$ should not be affected by the length of coexisting polymer 1 if $N_{\mathrm{e}} \ll N_{2}<N_{1}$.

\section{Concentration Dependence}

A simple consideration shows that $N_{\mathrm{e}}$ should be inversely proportional to $c_{\mathrm{p}}$; i.e.,

$$
N_{\mathrm{e}}=N_{\mathrm{e}}^{0} \rho c_{\mathrm{p}}{ }^{-1}
$$

where $N_{\mathrm{e}}^{0}$ is the value of $N_{\mathrm{e}}$ for the undiluted system and $\rho$ the density of the same system. With eq 19 , eq 9 gives

$$
D_{\mathrm{s}}{ }^{-1} \propto \zeta_{0}\left(c_{\mathrm{p}}\right) c_{\mathrm{p}}{ }^{3 / 2} N^{5 / 2}
$$

which predicts that $D_{\mathrm{s}}$ of a sufficiently high molecular weight monodisperse polymer in well-entangled solutions should vary linearly with $c_{\mathrm{p}}{ }^{-1.5}$ when it is corrected for $\zeta_{0}\left(c_{\mathrm{p}}\right)$.

Using the reptation model, Doi and Edwards $^{4}$ derived the relation

$$
D_{\mathrm{s}}{ }^{-1} \propto c_{\mathrm{p}}{ }^{2 \alpha+\beta} N^{2}
$$

where $\alpha$ is a parameter determining the concentration dependence of the tube radius $a$ :

$$
a \propto c_{\mathrm{p}}{ }^{-\alpha}
$$

and $c_{\mathrm{p}}{ }^{\beta}$ may be taken as representing the concentration dependence of $\zeta_{0}$. The scaling argument ${ }^{5}$ predicts $\alpha$ to be $3 / 4$, which gives 1.5 for $2 \alpha$. Thus, according to the Doi-Edwards theory combined with the scaling argument, $D_{\mathrm{s}}$ corrected for $\zeta_{0}$ should vary in proportion to $c_{\mathrm{p}}{ }^{-1.5}$. Although this prediction agrees with that from our theory, it is obvious that the agreement is only accidental.

Recently, Nemoto et al. ${ }^{6}$ found that $\zeta_{0^{-}}$ corrected $D_{\mathrm{s}}$ of polystyrene in tetrahydrofuran at high concentrations, i.e., in entangled solutions, depended linearly on $c_{\mathrm{p}}{ }^{-3}$. Our prediction by eq 20 does not conform to this result, but is fairly well consistent with another recent work on the same polymer+solvent system by von Meerwall et al., ${ }^{7}$ who showed $\zeta_{0}$-corrected $D_{\mathrm{s}}$ to vary linearly with $c_{\mathrm{p}}{ }^{-1.75}$. It is disturbing to see such distinctly different conclusions being reported on the same system. These groups of authors analyzed the data for entangled solutions using the $M^{-2}$ scaling predicted by reptation theory, ${ }^{1}$ and it appears from their papers that this treatment was successful. However, as shown in the Appendix, the data of von Meerwall et al. do not always warrant the use of $M^{-2}$ scaling.

The consideration leading to eq 19 also gives

$$
N_{\mathrm{e} 1}=N_{\mathrm{e}}^{0} \rho\left(c_{\mathrm{p}} w\right)^{-1}
$$

where $w$ is the weight fraction of polymer 1 in the blend of polymers 1 and 2. Using eq 19 and 23 , we obtain from eq 16

$$
D_{\mathrm{s} 1}{ }^{-1} \propto \zeta_{0}\left(c_{\mathrm{p}}\right) c_{\mathrm{p}}{ }^{3 / 2} N_{1} N_{\mathrm{w}}{ }^{3 / 2}
$$

where $N_{\mathrm{w}}$ is the weight-average chain length of the polymers constituting the blend under consideration; i.e.,

$$
N_{\mathrm{w}}=w N_{1}+(1-w) N_{2}
$$

Equation 24 predicts that $N_{1} D_{\mathrm{s} 1}$ at fixed $c_{\mathrm{p}}$ should vary in proportion to $N_{w}{ }^{-1.5}$. Experimental tests of this prediction serve to check the fundamental assumptions of the present theory, especially Assumption 3.

Finally, with eq 19 it follows from eq 18 that

$$
D_{\mathrm{s} 2}{ }^{-1} \propto \zeta_{0}\left(c_{\mathrm{p}}\right) c_{\mathrm{p}}{ }^{3 / 2} N_{2}{ }^{5 / 2}
$$

\section{DISCUSSION}

\section{Comparison with Experiment}

For very small values of $w$, eq 24 reduces to

$$
D_{\text {s1 }}{ }^{-1} \propto N_{2}{ }^{3 / 2} N_{1} \quad\left(N_{2}<N_{1}\right)
$$

The corresponding expression of $D_{\mathrm{s} 1}{ }^{-1}$ for $N_{2}>N_{1}$ can be obtained from eq 26 by ex- 


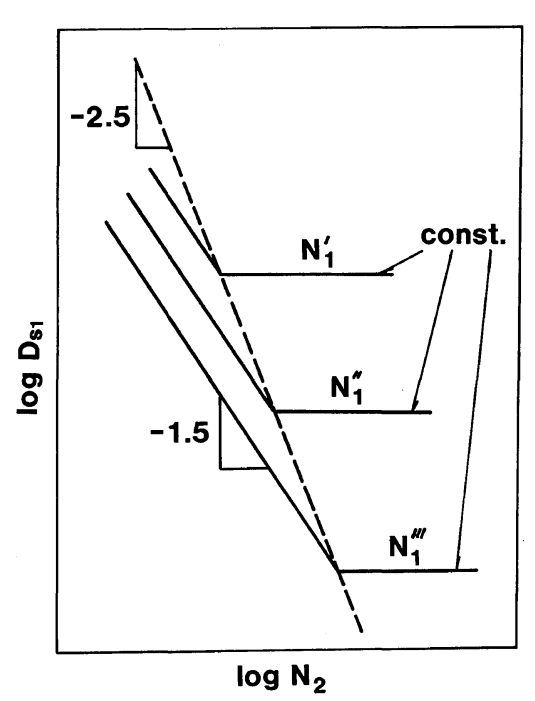

Figure 3. Theoretical prediction for self-diffusion coefficient $D_{\mathrm{s} 1}$ of the minor component 1 in binary polymer blends as a function of $N_{2}$ for fixed $N_{1}\left(N_{1}^{\prime}<N_{1}^{\prime \prime}<N_{1}^{\prime \prime}{ }^{\prime \prime}\right)$.

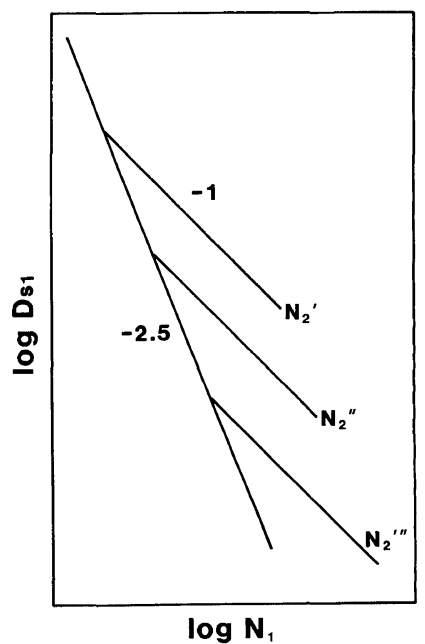

Figure 4. Theoretical prediction for self-diffusion coefficient $D_{\mathrm{s} 1}$ of the minor component 1 in binary polymer blends as a function of $N_{1}$ for fixed $N_{2}\left(N_{2}^{\prime}<N_{2}^{\prime \prime}<N_{2}^{\prime \prime \prime}\right)$.

changing the subscripts 1 and 2. Thus

$$
D_{\text {s1 }}{ }^{-1} \propto N_{1}{ }^{5 / 2} \quad\left(N_{1}>N_{2}\right)
$$

The proportionality factors in eq 27 and 28 are the same (a function of $c_{\mathrm{p}}$ and $T$ ), because both equations should agree at $N_{1}=N_{2}$.

Figures 3 and 4 illustrate, respectively, $D_{\mathrm{s} 1}$ at fixed $N_{1}$ as a function of $N_{2}$ and $D_{\mathrm{s} 1}$ at fixed $N_{2}$ as a function of $N_{1}$, calculated from eq 27 and 28. It can be seen from Figure 3 that, for any fixed $N_{1}, D_{\mathrm{s} 1}$ decreases in proportion to $N_{2}^{-1.5}$ as $N_{2}$ increases to $N_{1}$ and then becomes constant for $N_{2}$ above $N_{1}$. The dashed line connecting the break points has a slope of -2.5 , indicating that $D_{\mathrm{s}}$ for a monodisperse polymer in well-entangled regions is a linear function of $M^{-2.5}$. Figure 4 indicates that $D_{\text {s1 }}$ for fixed $N_{2}$ decreases in proportion to $N_{1}^{-1.0}$ for $N_{1}$ above $N_{2}$. The locus of the break points, which has a slope of -2.5 , gives the molecular weight dependence of $D_{\mathrm{s}}$ of a monodisperse polymer in well entangled regions. It should be noted that these results are concerned with values of $N_{1}$ and $N_{2}$ much larger than $N_{\mathrm{e}}$.

Diffusion data on binary polymer blends with $w \ll 1$ were obtained by Tanner $^{8}$ on poly(dimethylsiloxane), by Bueche $^{9}$ and Kumagai et $a t .^{10}$ on polystyrene, and by Klein ${ }^{11}$ on polyethylene. Here, the data of Kumagai et al. ${ }^{10}$ are compared with our theoretical predictions, because they are consistent with the above-mentioned condition for $N_{1}$ and $N_{2}$; most of Tanner's data are concerned with $N_{1}<N_{\mathrm{c}}$, and Bueche's data as well as Klein's only with $N_{\mathrm{e}}<N_{1}<N_{2}$, where $N_{\mathrm{c}}$ is $N$ characterizing the onset of chain entanglement.

Figures 5 and 6 show the data of Kumagai et al. corresponding to Figures 3 and 4 . Although the scatter of data points is considerable, we can appreciate overall similarity between Figures 3 and 5 . The data points for each fixed $M_{1}$ in the region of $M_{2}$ below $M_{1}$ are approximately fitted by a straight line of slope -1.5 (Kumagai et al. ${ }^{10}$ assigned -1.7 to this slope), in agreement with the consequence from our theory. Except for the lowest $M_{1}$ $\left(1.10 \times 10^{5}\right), D_{\mathrm{s} 1}$ appears to level off rapidly as $M_{2}$ exceeds a given $M_{1}$. The horizontal part of each curve in Figure 3 may be regarded as an idealization of this behavior. A similar insensitivity of $D_{\mathrm{s} 1}$ to $M_{2}$ in the region of $M_{2}$ 


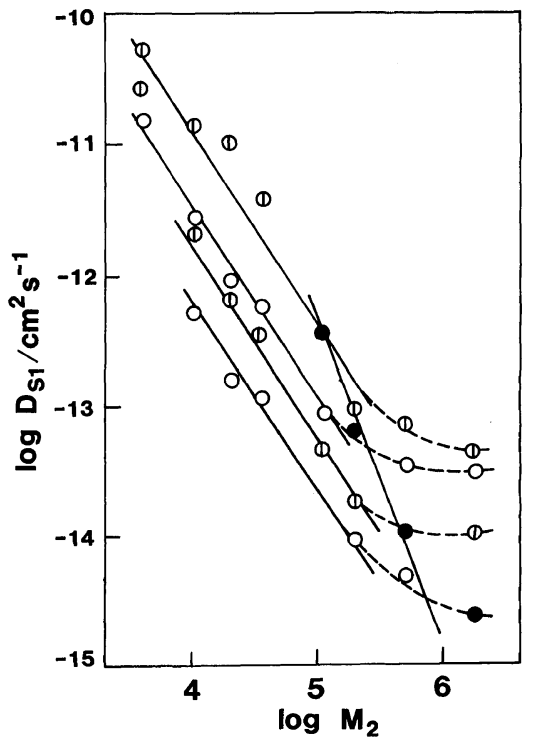

Figure 5. Self-diffusion coefficient $D_{\mathrm{s} 1}$ of the minor component $M_{1}$ in binary polystyrene mixtures at $150^{\circ} \mathrm{C}$ as a function of $M_{2}$ of the matrix component. $M_{1}$ is $1.10 \times 10^{5}, 2.00 \times 10^{5}, 4.98 \times 10^{5}$, and $1.80 \times 10^{6}$ from top to bottom. Filled circles indicate $D_{\mathrm{s}}$ for $M_{1}=M_{2}$, and the straight line fitting them is drawn with slope -2.5 . The four straight lines fitting data points for $M_{2}<M_{1}$ have a slope of -1.5 .

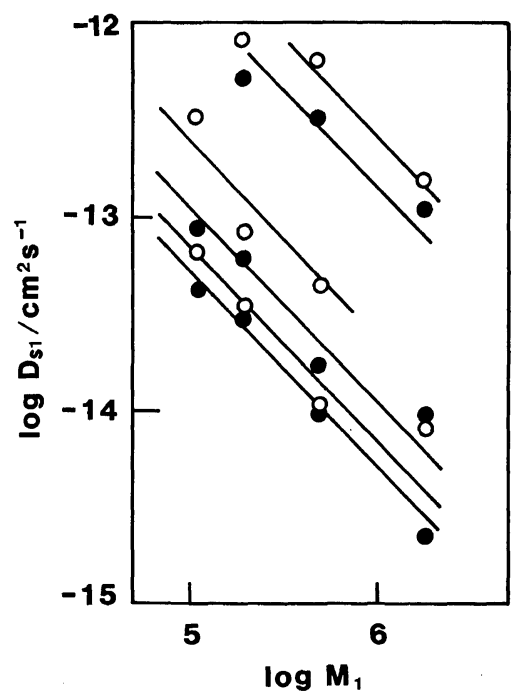

Figure 6. Self-diffusion coefficient $D_{\mathrm{s} 1}$ of the minor component $M_{1}$ in binary polystyrene mixtures at $150^{\circ} \mathrm{C}$ for fixed molecular weight $M_{2}$ of the matrix component. $M_{2}$ is $2.04 \times 10^{4}, 3.70 \times 10^{4}, 1.10 \times 10^{5}, 2.00 \times 10^{5}$, $4.98 \times 10^{5}$, and $1.80 \times 10^{6}$ from top to bottom. The straight lines are drawn with slope -1 . above $M_{1}$ is seen in the data of Tanner, ${ }^{8}$ Bueche, ${ }^{9}$ and Klein. ${ }^{11}$

The filled circles in Figure 5 indicate the $D_{\mathrm{s} 1}$ values for $M_{2}=M_{1}$. The line fitting the upper three of them has a slope of -2.5 , thus yielding $D_{\mathrm{s}} \propto M^{-2.5}$ for a monodisperse entangled system as predicted by eq 9 .

The straight lines in Figure 6 were drawn with slope of -1 so as to be consistent with the behavior of the curves at the left to the breaks in Figure 4. However, the data reveal no theoretically predicted linear part with slope -2.5 .

\section{Concentration Dependence of $N_{\mathrm{e}}$}

The conventional method for estimating $N_{\mathrm{e}}$ in entangled polymer concentrates is to utilize plateau moduli determined by viscoelastic measurements. Typical data on $N_{\mathrm{e}}$ have been fitted by an empirical relation ${ }^{12}$

$$
N_{\mathrm{e}} \propto c_{\mathrm{p}}^{-\delta}
$$

with $\delta$ ranging $1.0-1.3$. This is not in accord with eq 19 deduced on a simple consideration. Assuming that mesh chains between neighboring entanglements in semidilute solutions of a flexible polymer are expanded by volume exclusion to a maximum degree, de Gennes ${ }^{1,5}$ showed $\delta$ to be 1.25 , which is consistent with the experimental values mentioned above. However, it is less likely that his assumption holds for well-entangled systems, in which excluded-volume effect is known to virtually vanish.

\section{Concluding Remarks}

In this paper, we have developed a theory of self diffusion in highly entangled polymer solutions by considering free wriggling to be more realistic chain motion in such systems than reptation and incorporating the noodle effect approximately into the formulation. This effect was once the central subject in the dynamics of polymer concentrates, ${ }^{13}$ but it seems to have been unduly ignored in recent publications. We maintain that it plays a cru- 
cial role in determining dynamical behavior of polymer in concentrated solutions and melts and should be brought back to the stage of current discussion.

Our theory described in this paper is essentially concerned with the asymptotic behavior of polymer chains sufficiently entangled with other chains so that the condition $N / N_{\mathrm{e}} \gg 1$ is satisfied. This condition facilitates calculating the number of entangling chains per polymer molecule. A more elaborate treatment would become necessary for formulating the noodle effect on less densely entangled chain for which $N / N_{\mathrm{e}}$ is comparable to unity.

\section{APPENDIX}

Figure 7 shows $\log -\log$ plots of $D_{\mathrm{s}}$ vs. $c_{\mathrm{p}}$ at fixed $M$ constructed using the data listed in Table II of von Meerwall et al.'s paper. ${ }^{7}$ The arrows indicate the $c_{\mathrm{p}}$ values above which the

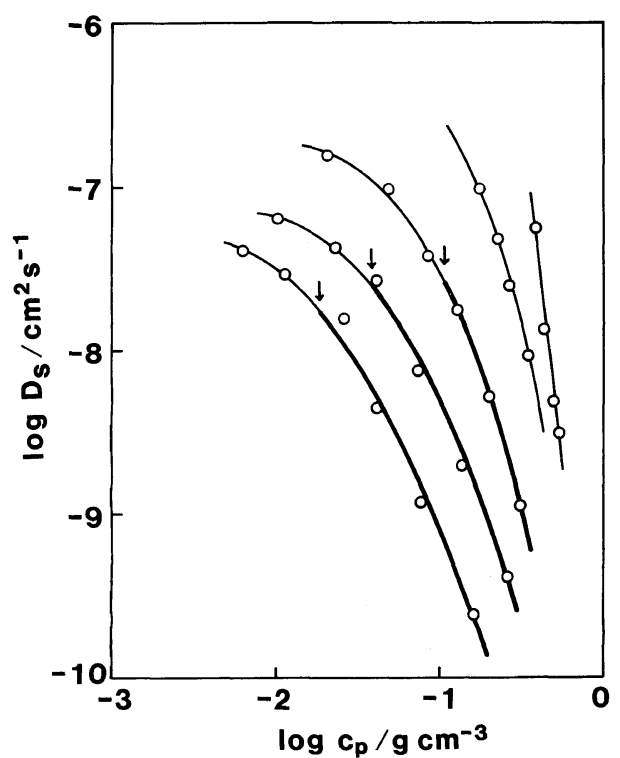

Figure 7. Concentration dependence of self-diffusion coefficient $D_{\mathrm{s}}$ of polystyrene in tetrahydrofuran at $30^{\circ} \mathrm{C}$. The molecular weight is $1.05 \times 10^{6}, 4.98 \times 10^{5}, 1.79 \times 10^{5}$, $3.74 \times 10^{4}$, and $1.0 \times 10^{4}$ from left to right. Arrows indicate the critical concentrations $c_{\mathrm{c}}$ calculated by $c_{\mathrm{e}}=$ $\rho M_{\mathrm{e}}^{\circ} / M_{\mathrm{w}}$ with $\rho=1.05 \mathrm{~g} \mathrm{~cm}^{-3}$ and $M_{\mathrm{e}}^{\circ}=18100$. solution becomes chain entangled. The values of $\log D_{\mathrm{s}}$ at four fixed $c_{\mathrm{p}}$ interpolated or extrapolated from the smooth curves in Figure 7 are plotted against $\log M$ in Figure 8 . Here, each arrow indicates the $M$ value for the onset of entanglement at a given $c_{\mathrm{p}}$, and the dashed lines are drawn with slopes of -2.0 and -2.5 . Any of the smooth curves in Figure 8 clearly shows downward curvature, with its slope changing continuously from -1.5 toward -2.5 with increasing $M$. Thus, we find that the data of von Meerwall et al. do not obey the $M^{-2}$ scaling even in entangled solutions, but rather appear to support the inference from our recent data analysis ${ }^{3}$ as well as the prediction of the present theory that $D_{\mathrm{s}}$ in polymer concentrates becomes asymptotically proportional to $M^{-2.5}$.

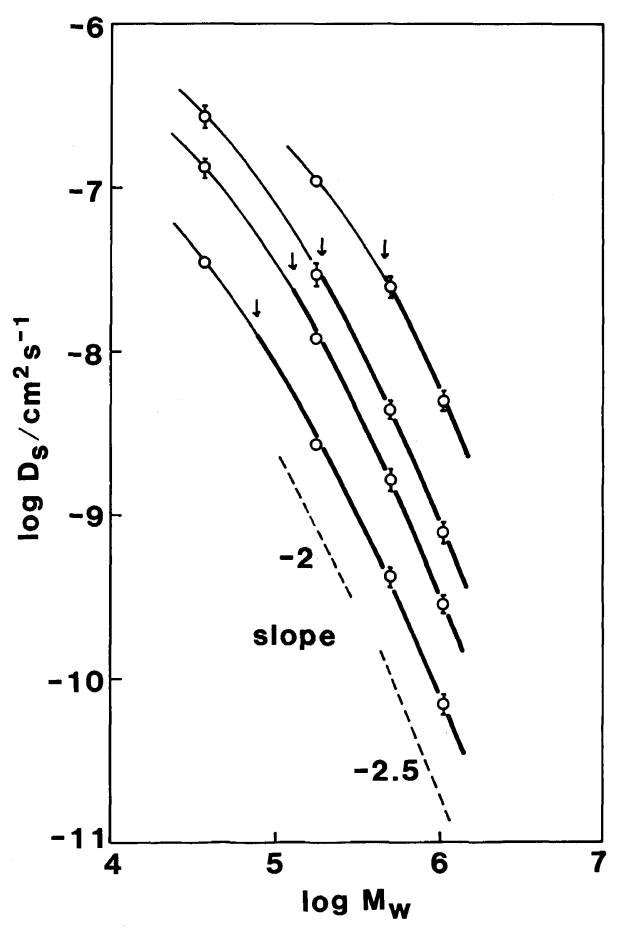

Figure 8. Molecular weight dependence of selfdiffusion coefficient $D_{\mathrm{s}}$ of polystyrene in tetrahydrofuran at $30^{\circ} \mathrm{C}$ for fixed concentration $c_{\mathrm{p}} \cdot c_{\mathrm{p}}$ is $0.04,0.10,0.15$, and $0.25 \mathrm{~g} \mathrm{~cm}^{-3}$ from top to bottom. Arrows indicate the critical molecular weights $M_{\mathrm{e}}$ calculated by $M_{\mathrm{e}}=$ $\rho M_{\mathrm{e}}^{\circ} / \mathcal{C}_{\mathrm{p}}$. 


\section{Self Diffusion}

\section{REFERENCES}

1. P. G. de Gennes, "Scaling Concepts in Polymer Physics," Cornell University Press, Ithaca, N. Y., 1979.

2. M. Tirrell, "Polymer Self Diffusion in Entangled Systems," Rubber Technol. Chem., 57, 523 (1984).

3. Y. Einaga and H. Fujita, Reoroji Gakkaishi, 12, 136 (1984).

4. M. Doi and S. F. Edwards, J. Chem. Soc., Faraday. Trans. 2, 74, 1789 (1978).

5. P. G. de Gennes, Macromolecules, 9, 594 (1976).

6. N. Nemoto, M. R. Labdry, I. Noh, T. Kitano, J. A.
Wesson, and H. Yu, Macromolecules, 18, 308 (1985).

7. E. D. von Meerwall, E. J. Amis, and J. D. Ferry, Macromolecules, 18, 206 (1985).

8. J. E. Tanner, Macromolecules, 4, 748 (1971).

9. F. Bueche, J. Chem. Phys., 48, 1410 (1968).

10. Y. Kumagai, H. Watanabe, K. Miyasaka, and T, Hata, J. Chem. Eng. Jpn., 12, 1 (1979).

11. J. Klein, Phil. Mag., A43, 771 (1981).

12. V. R. Raju, E. V. Menezes, W..W. Graessley, and L. J. Fetters, Macromolecules, 14, 1668 (1981); K. Osaki, H. Nishizawa, and M. Kurata, Reoroji Gakkaishi, 10, 169 (1982).

13. W. W. Graessley, Adv. Polym. Sci., 16, 1 (1974). 